\title{
Desmutagenic Effect of Pheophytin b against Trp-P-2 in the Salmonella Assay
}

\author{
Kentaro Yoshikawa, ${ }^{1}$ Pramod Aryal, ${ }^{1}$ Takao Terashita, ${ }^{1}$ Jiko Sishiyama ${ }^{1}$ and Delbert M. Shankel ${ }^{2}$ \\ ${ }^{1}$ Department of Food and Nutrition, Kin-ki University, 3327-204, Nakamachi, Nara 631, Japan \\ ${ }^{2}$ Department of Microbiology, The University of Kansas, Lawrence, KS 66049, USA
}

Received July 2, 1997; Accepted October 13, 1997

\begin{abstract}
Modifications of the standard Ames test and $u m u$ test were employed to study the "desmutagenic" response exerted by pheophytin b against the mutagenicity of 3-amino-1-methyl-5H-pyrido[4,3-b] indole (Trp-P-2). The mixture from which pheophytin b separated was activated with $\mathrm{S} 9 \mathrm{mix}$ and was assayed to determine the remaining mutagenicity of Trp-P-2. The decrease in the $\mathrm{His}^{+}$revertant colonies of Salmonella typhimurium TA98 was dependent on the increase in pheophytin b concentrations. Binding of Trp-P-2 to DNA and the subsequent induction of the SOS response were investigated using the umu test employing Salmonella typhimurium NM 2009. Trp-P-2 induced induction of $\beta$-galactosidase was suppressed when pheophytin b was included in the test. In order to evaluate the mode of action of pheophytin $b$ in the mixture of Trp-P-2, Trp-P-2 was analyzed using HPLC. Compared with a solution of Trp-P-2 alone, the absorbance peak of Trp-P-2 in the HPLC profile of the mixture of Trp-P-2 and pheophytin b was observed to be decreased. Also a new peak was not shown in the HPLC profile. To investigate the possible effect of pheophytin b on the S9 mix, the HPLC profile of a mixture containing Trp-P-2, S9 mix and pheophytin b was compared with a mixture containing only Trp-P-2 and S9 mix. The absorbance peak of the hydroxylated Trp-P-2 in the reaction mixture containing pheophytin $b$ was observed to be smaller than the peak of the mixture without pheophytin $b$. From these results, it is suggested that pheophytin b limits the formation of hydroxylated Trp-P-2.
\end{abstract}

Keywords: desmutagenicity, pheophytin, Salmonella typhimurium TA98, Salmonella typhimurium NM2009, Ames test, umu test

Many investigations have been carried out to identify naturally occurring and synthetic compounds capable of suppressing mutagenicity and carcinogenicity. Several literature reports point out the existence of compounds in daily food (Yoshikawa et al., 1985; Yoshikawa \& Ishii, 1987) which are capable of suppressing the formation of mutagens or carcinogens and/or are capable of inactivating mutagens or carcinogens. The factors which inactivate mutagens or carcinogens, particularly those from vegetables, fruits and dietary materials of plant origin, have been considered to exhibit important inhibitory effects on the development of human cancer. However, the exact mechanisms of inactivation of mutagens or carcinogens are not yet sufficiently understood.

High heat treatment or cooking of food materials rich in protein results in the formation of mutagenic heterocyclic amines with high potential for carcinogenicity (Sugimura et al., 1977). Morita et al. (1978) have reported the suppression of heterocyclic amines induced mutagenesis by vegetables such as cabbages, broccoli, eggplants and others in the Salmonella/microsome assay. These extracts have been demonstrated to suppress the effects of heterocyclic amines such as 3-amino-1,4-dimethyl-5 $H$-pyrido [4,3-b] indole (Trp$\mathrm{P}-1)$ as shown by induced mutagenesis using the Ames test. Peroxidase in cabbage was identified by Inoue et al. (1981) as an agent which plays an important role. Yoshikawa et al. (1990) found that the mutagenicity of broiled fish was inhibited by crude extracts of vegetables such as burdock, eggplant, broccoli and others. Japanese eggplant extract has been demonstrated to suppress 3 -amino-1-methyl-5 $H$-pyrido [4,3-b] indole (Trp-P-2) induced mutagenesis in Salmonella typhimurium TA98 (Yoshikawa et al., 1996a), and one of the mutation-suppressing compounds was identified as pheophytin (Yoshikawa et al., 1996b).

The present paper deals with the desmutagenic mechanism of mutation suppression by pheophytin $b$, a plant porphyrin.

\section{Materials and Methods}

Bacterial strains and chemicals The histidine-requiring strain of S. typhimurium TA98 was generously provided by Dr. T. Kada (National Institute of Genetics, Mishima). The S. typhimurium NM2009 used to study induction of the umu operon was a generous gift from Dr. Y. Oda (Osaka Prefectural Institute of Public Health, Osaka).

The chemicals used in this study were from the following sources: the $\mathrm{S} 9$ was prepared from Sprague-Dawley male rats treated with phenobarbital and benzoflavone, and the cofactors were purchased from the Oriental Yeast Co., Tokyo; 3-amino-1-methyl-5 $H$-pyrido [4,3- $b$ ] indole (Trp-P-2), pheophytin $b$ from chlorella were purchased from Wako Pure Chemical, Osaka. All other chemicals and reagents used were of the highest quality commercially available.

Ames test The desmutagenic effect of pheophytin b was assayed using $S$. typhimurium TA98. The reaction mixture, adjusted to $1.0 \mathrm{ml}$ by the addition of phosphate buffer $\left(\mathrm{pH} 7.0,37^{\circ} \mathrm{C}\right)$, containing $10 \mathrm{nmol}$ of Trp-P-2 and 
different concentrations of pheophytin b dissolved in dimethyl sulfoxide (DMSO), was allowed to react for $30 \mathrm{~min}$ in air at $37^{\circ} \mathrm{C}$ with gradual shaking. The reaction mixture was then heated at $100^{\circ} \mathrm{C}$ for $3 \mathrm{~min}$. The mixture was filtered using membrane filters $(0.45 \mu \mathrm{m})$, and the filtrate was assayed. The assay procedure was as described in Mitscher et al. (1986), Shankel and Clarke (1990) and Yoshikawa et al. (1991). The mutagen used requires S9 for metabolic activation. The mutagenicity of the mutagen in the absence of pheophytin $b$ is defined as $100 \%$ remaining mutagenicity. Each set of experiments was run in triplicate and repeated at least 3 times.

Umu test The umu test was carried out as described by Oda et al. with slight modification using $S$. typhimurium NM 2009 (Oda et al. 1985, 1995). The bacterial strain was grown in Luria broth medium containing ampicillin $(25 \mu \mathrm{g} / \mathrm{ml})$ at $37^{\circ} \mathrm{C}$ for $16 \mathrm{~h}$ with shaking. The overnight culture was transferred to TGA medium ( $1 \%$ Bactotryptone, $0.5 \% \mathrm{NaCl}$, $0.2 \%$ glucose, $20 \mathrm{mg} / \mathrm{ml}$ ampicillin) at a 50 -fold dilution and was then cultured at $37^{\circ} \mathrm{C}$ till the absorbance at $600 \mathrm{~nm}$ reached 0.3 . In another test tube, $1.7 \mathrm{ml}$ of this culture, $0.3 \mathrm{ml}$ of S9 mix, $10 \mu \mathrm{l}$ of Trp-P-2 $(1 \mu \mathrm{g} / \mathrm{ml})$ and $10 \mu \mathrm{l}$ of pheophytin $b$ (of different concentrations) were added, and the mixture was incubated for $2 \mathrm{~h}$ at $37^{\circ} \mathrm{C}$ with shaking for the induction of the $u m u$ operon. The bacterial density at $600 \mathrm{~nm}$ was measured, and $\beta$-galactosidase activity was assayed using 2 -nitrophenyl- $\beta$-D-galactopyranoside as the substrate. The activity was determined by the method of Miller (1972) as modified by Oda et al. (1985).

HPLC analysis in the mixture of Trp-P-2 and pheophytin $b$ In the reaction mixture of $1.0 \mathrm{ml}, 0.1 \mathrm{ml}$ each of $0.2 \mathrm{mM}$ Trp-P-2 (in water) and $0.4 \mathrm{mM}$ pheophytin b (in DMSO) were added in a phosphate buffer, $\mathrm{pH}$ 7.0. The reaction was allowed to proceed for $30 \mathrm{~min}$ at $37^{\circ} \mathrm{C}$ and was stopped by the addition of $1.0 \mathrm{ml}$ cold acetone. The reaction mixture was then centrifuged $\left(8000 \times g, 4^{\circ} \mathrm{C}\right.$ for $\left.10 \mathrm{~min}\right)$. The supernatant was evaporated under reduced pressure at $36^{\circ} \mathrm{C}$ using a rotary evaporator until completely dry. The dried residue was then dissolved in $1.0 \mathrm{ml}$ of $50 \mathrm{~mm}$ disodium phosphate- $25 \mathrm{~mm}$ citrate buffer, $\mathrm{pH} 4.0$. The solution was diluted 10 times, and $20 \mu \mathrm{l}$ was employed for HPLC analysis. The result of the HPLC analysis was shown by the absorbance at UV $254 \mathrm{~nm}$ (Arimoto \& Hayatsu, 1989) using a Shimadzu 10AS liquid chromatography apparatus with a Wakosil 5 C18 $(4.6 \phi \times 200 \mathrm{~mm})$ column. The mobile phase used was acetonitrile and ammonium sulfate $(1: 3, \mathrm{v} / \mathrm{v})$ with a flow rate of $1.0 \mathrm{ml} / \mathrm{min}$.

Effect of pheophytin $b$ on $N$-hydroxylation of Trp-P-2 The $N$-hydroxylation of Trp-P-2 was investigated by analyzing the HPLC profile by a method similar to that as described for the HPLC analysis of the mixture of Trp-P-2 and pheophytin $b$. To this mixture, $0.5 \mathrm{ml}$ of $\mathrm{S} 9$ mix was added prior to the incubation, and the peak corresponding to the $N$-hydroxy Trp-P-2 was analyzed.

\section{Results}

Ames test Using S. typhimurium TA98, the mutationsuppressing ability of pheophytin $\mathrm{b}$ was assayed by the Ames test. The remaining mutagenicity of Trp-P-2 on co-incubating pheophytin $b$ is shown in Fig. 1. The addition of $3.0 \mathrm{nmol}$ of pheophytin $b$ to the test plate resulted in $36 \%$ suppression of the mutagenicity induced by $10 \mathrm{nmol}$ of Trp-P-2, and $39 \%$ suppression was recognized when $4.0 \mathrm{nmol}$ of pheophytin $\mathrm{b}$ was added. Further increases in the concentration of pheophytin b did not result in any more suppression: addition of $8.0 \mathrm{nmol}$ resulted in $40 \%$ inhibition.

Umu test Using S. typhimurium NM2009, an investigation was undertaken to demonstrate that pheophytin $b$ prevents the binding of the ultimate mutagenic metabolite of Trp-P-2 to the DNA molecule which then induces the $u m u$ operon. The Trp-P-2 induced levels of $\beta$-galactosidase in the $S$. typhimurium NM2009 were confirmed to decrease as a result of the addition of pheophytin $b$. The $\beta$-galactosidase activity was observed to decrease by $23 \%$ on co-incubating Trp-P-2 with $1.5 \mathrm{nmol} / \mathrm{ml}$ of pheophytin b (Fig. 2). Further increases in the concentration of pheophytin $b$ seem to slightly increase the $\beta$-galactosidase activity; however, the $\beta$-galactosidase activity remained lower than the value attained without pheophytin $b$. Higher concentrations of pheophytin b could not be employed in the test because of the disturbances of green color on the absorbance at $420 \mathrm{~nm}$ and $550 \mathrm{~nm}$. These results indicate that the decrease in $\beta$-galactosidase activity is due to a decrease in the induction of the

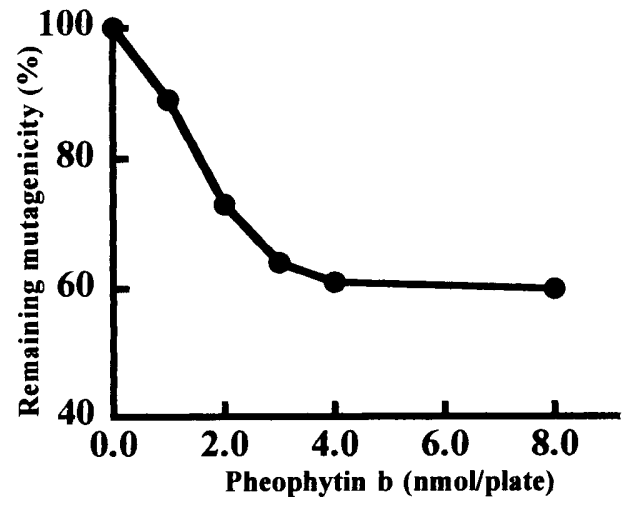

Fig. 1. Effect of pheophytin b on Trp-P-2 (10 nmol/plate) induced mutagenesis in Salmonella typhimurium TA98. Mean colony counts \pm standard deviation of Trp-P-2 (10 nmol/plate)-induced $\mathrm{His}^{+}$revertants were $1462 \pm 91$ $(n=9)$, and mean colony counts \pm standard deviation of spontaneous $\mathrm{His}^{+}$ revertants were $25 \pm 10(n=9)$.

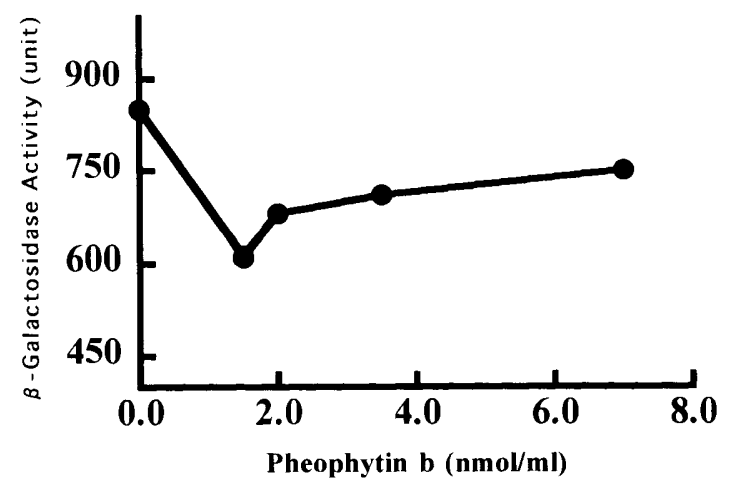

Fig. 2. Effect of pheophytin b on Trp-P-2 (50 ng) induced induction of $u m u$ operon in Salmonella typhimurium NM2009. 


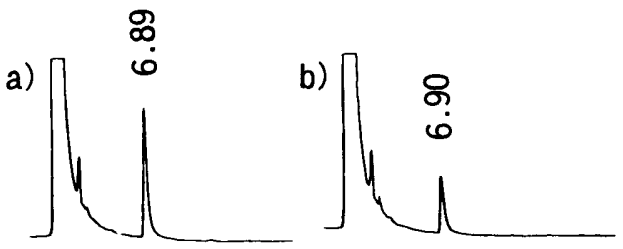

Fig. 3. HPLC profiles of the Trp-P-2 reaction mixture. a: without pheophytin $b$, b: with pheophytin $b$. Trp-P-2 $(20 \mathrm{nmol})$ was incubated with $0.4 \mathrm{mM}$ pheophytin $b$ at $37^{\circ} \mathrm{C}$ for $30 \mathrm{~min}$ and then analyzed on HPLC as mentioned in the text. The peak at $6.89(6.90)$ retention time corresponds to Trp-P-2.
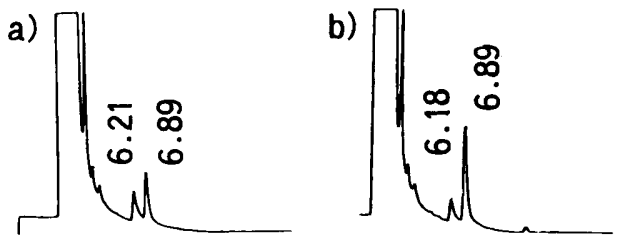

Fig. 4. HPLC profiles of the S9-mediated hydroxylation reaction mixture. a: without pheophytin $b$, $b$ : with pheophytin $b$. Trp-P-2 $(20 \mathrm{nmol})$ was incubated with $0.4 \mathrm{~mm}$ pheophytin $\mathrm{b}$ and $0.5 \mathrm{ml} \mathrm{S} 9 \mathrm{mix}$ at $37^{\circ} \mathrm{C}$ for $30 \mathrm{~min}$ and then analyzed on HPLC as mentioned in the text. The peaks at $6.18(6.21)$ and 6.89 retention times correspond to $N$-hydroxy Trp-P-2 and Trp-P-2, respectively.

umu operon and confirm that pheophytin b can suppress mutability.

HPLC analysis of the mixture of Trp-P-2 and pheophytin $b$ To ascertain whether or not pheophytin $b$ reacted directly with Trp-P-2, the remaining Trp-P-2 in solution was estimated by HPLC. It was recognized that compared to the HPLC peak of $2.0 \mathrm{nmol}$ Trp-P-2 alone at retention time (Rt) 6.90 (Fig. 3a), the HPLC peak of Trp-P-2 reacted with pheophytin $\mathrm{b}$ at Rt 6.89 drastically decreased (Fig. 3b). This result indicates that the amount of free Trp-P-2 molecules in the reaction mixture containing pheophytin $b$ decreases by a considerable amount.

Effect of pheophytin $b$ on $N$-hydroxylation of Trp-P-2 To confirm the $N$-hydroxylation of Trp-P-2 resulting from reactions with the S9 mix, HPLC analysis of the mixture of Trp-P-2, S9 and pheophytin b was performed. The HPLC profile at $254 \mathrm{~nm}$ of the reaction mixture containing Trp-P-2 and the S9 mix exhibited a peak at Rt 6.21 min for the $N$-hydroxy metabolite of Trp-P-2, and a peak at Rt $6.89 \mathrm{~min}$ for Trp-P-2 alone (Fig. 4a), as reported by Arimoto et al. (1989). When $42.2 \mathrm{nmol}$ of pheophytin $\mathrm{b}$ was added to the mixture containing 20 nmol of Trp-P-2, shown in Fig. 4b, the peak for Trp-P-2 (Rt, 6.89) produced a greater peak area and the peak for the $N$-hydroxy metabolite of Trp-P-2 (Rt, 6.18) produced a smaller peak area, compared to the reaction mixture without pheophytin $b$. The comparatively small peak area for the $N$-hydroxy metabolite of Trp-P-2 in the reaction mixture with pheophytin $b$ indicates a decrease in the enzymatic formation of the $N$-hydroxy metabolite.

\section{Discussion}

From the cancer-chemoprevention point of view, it is considered that the mutation-suppressing compounds in our daily diet may deter the onset of cancer. This idea is based on the isolation and identification of mutation-suppressing compounds from vegetables and fruits and the epidemiological data showing a correlation between dietary habits and susceptibility to carcinogenesis (Block et al., 1992).

In earlier reports, it was demonstrated that Japanese eggplant extracts suppressed the mutagenicity induced by some mutagenic heterocyclic amines, and one of the compounds exhibiting desmutagenicity towards Trp-P-2 induced mutagenicity was identified as pheophytin (Yoshikawa et al., 1996b). In the present study, the desmutagenicity of pheophytin b was investigated employing the umu test, and the speculative desmutagenic mechanism was investigated.

After the reaction mixture of Trp-P-2 and pheophytin b was heated and filtered, the filtrate was activated with the S9 mix, and the mixture was assayed to determine the remaining mutagenicity of Trp-P-2 using $S$. typhimurium TA98. The decrease in the $\mathrm{His}^{+}$revertant colonies depends on the pheophytin b concentration from $1.0 \mathrm{nmol}$ to $3.0 \mathrm{nmol}$ per plate. The inhibition of $\mathrm{His}^{+}$revertant colonies was calculated to be about $40 \%$ on addition of more than $4.0 \mathrm{nmol}$ of pheophytin b per test plate (Fig. 1). Chlorophyllin, a manmade salt of chlorophyll containing either sodium or copper as the central chelating metal, has been observed to inhibit the mutagenic activity of amino- $\alpha$-carboline, 2-amino-6-methyldipyrido $\left[1,2 \mathrm{a}: 3^{\prime}, 2^{\prime}-d\right]$ imidazol, 2-amino-6-dipyrido [1,2a:3',2'$d$ [imidazol, aminomethyl- $\alpha$-carboline, 3-amino-3,4-dimethyl-5 $H$-pyrido[4,3-b] indole, and Trp-P-2 in Salmonella/ microsome assay with TA98 in the presence of S9 (Arimoto et al., 1980, 1993; Dashwood et al., 1991; Dashwood \& Guo, 1993). In an earlier report, we observed that pheophytin b inhibits the mutagenicity of Trp-P-2 to a similar extent as that observed with chlorophyll in Salmonella/microsome assay with TA98 in the presence of S9 (Yoshikawa et al., 1996b).

Further more, the binding of the electrophilic metabolite of Trp-P-2 to DNA and the subsequent induction of the SOS response of $S$. typhimurium NM 2009 were investigated using the $u m u$ test. Trp-P-2 induced induction of $\beta$-galactosidase was recognized to be suppressed on addition of pheophytin $b$ in the test (Fig. 2). Slight increases in $\beta$-galactosidase activity were observed with increasing concentrations of pheophytin b. The exact cause of this effect is not clear, but it is assumed that the enhancing expression of $\beta$-galactosidase may be due to the green color of pheophytin b which shows the absorbance at $550 \mathrm{~nm}$. The decrease in $\beta$-galactosidase activity suggests that pheophytin $b$ inhibits the binding of the electrophilic metabolite of Trp-P-2 (acetylated form) to the DNA of $S$. typhimurium NM 2009, thus inhibiting the expression of the umu operon. This strain contains plasmid pSK 1002 having an umu C' lac Z fusion gene whose expression is regulated in the same manner as that of the umu operon (Okai et al., 1996).

From the results of the Ames and $u m u$ tests, it is suggested that pheophytin $b$ exhibits desmutagenicity towards Trp-P-2 induced mutagenesis by preventing the binding of the ultimate mutagenic metabolite of Trp-P-2 to the DNA. In order to further confirm the mechanism of desmutagenicity of pheophytin b, pheophytin b was allowed to react with 
Trp-P-2, and HPLC analysis of the reaction mixture was carried out. Compared to the control without pheophytin b, the UV $254 \mathrm{~nm}$ absorbance peak corresponding to Trp-P-2 decreased in the reaction mixture containing pheophytin $b$ (Fig. 3). The decrease in the absorbance peak indicated the decrease in free Trp-P-2 molecules. The absence of any additional new peak indicated that these two reacting molecules did not form a complex, as in the case of chlorophyll (Arimoto et al., 1993). We believe that these two molecules are stacked on each other and exhibit some sort of spatial arrangement which is not detected under the present analytical conditions.

It is known that promutagenic Trp-P-2 is enzymatically biotransformed to its hydroxylated form by microsomal protein (Hashimoto et al., 1980; Guengerich, 1988; Guengerich \& Shimada, 1991), and the hydroxylated metabolite is further metabolized to the ultimate mutagenic electrophilic metabolite (acetylated form) by $O$-acetyltransferase (Kato \& Yamazoe, 1988; Watanabe et al., 1990). From the results of HPLC analysis of the pheophytin b and Trp-P-2 reaction mixture, it is thought that the suppression of $\mathrm{His}^{+}$revertant colonies of S. typhimurium TA 98 and the reduced induction of the umu operon of S. typhimurium NM 2009 are due to the interception of Trp-P-2 by pheophytin $b$, hence inhibiting activation by $\mathrm{S} 9$. When we investigated the effects on the $\mathrm{S} 9$ mix mediated hydroxylation of Trp-P-2, the HPLC profile of the reaction mixture containing Trp-P-2, S9 mix and pheophytin $b$, the UV $254 \mathrm{~nm}$ absorbance peak associated with hydroxylated Trp-P-2 decreased, whereas the absorbance peak associated with Trp-P-2 alone increased, compared to that of the reaction mixture with Trp-P-2 and the S9 mix (Fig. 4). This clearly suggests that pheophytin $b$ inhibits the formation of hydroxylated the Trp-P-2 by the inhibition of S9 mix mediated hydroxylation.

Based on these studies, it is suggested that pheophytin $b$ exhibits desmutagenicity towards Trp-P-2 induced mutagenesis by limiting Trp-P-2 for the enzymatic metabolism to its hydroxy-form, most probably by intercepting Trp-P-2 molecules.

Acknowledgments The authors express their appreciation to $\mathrm{Mr}$ Yoshihisa Takada, Osaka Foundation of International Exchange (OFIX), for a part of the financial support and to Miss Keiko Shimizu and the colleagues at the Food Microbiology Laboratory for technical assistance.

\section{References}

Arimoto, S., Negishi, T. and Hayatsu, H. (1980). Inhibitory effect of hemin on the mutagenic activities of carcinogens. Cancer Lett., 11, 29-33.

Arimoto, S. and Hayatsu, H. (1989). Role of hemin in inhibition of mutagenic activity of 3-amino-1-methyl-5 $\mathrm{H}$-pyrido $[4,3-b]$ indole (Trp-P-2) and other aminoazarines. Mutat. Res., 213, 217-226.

Arimoto, S., Fukuoka, S., Itome, C., Nakano, H., Rai, H. and Hayatsu, H. (1993). Binding of polycyclic planar mutagens to chlorophyllin resulting in inhibition of the mutagenic activity. Mutat. Res., $\mathbf{2 8 7}$ 293-305.

Block, G., Patterson, B. and Subari, A. (1992). Fruits, vegetables and cancer prevention : a review of the epidemiological evidence. Nutr. Cancer, 18, 1-29.

Dashwood, R.H., Breinholt, V. and Bailey, G.S. (1991). Chemopreventive properties of chlorophyllin: inhibition of aflatoxin $B_{1}$
$\left(\mathrm{AFB}_{1}\right)$-DNA binding in vivo and anti-mutagenic activity against $\mathrm{AFB}_{1}$ and two heterocyclic amines in the Salmonella mutagenicity assay. Carcinogenesis, 12, 939-942.

Dashwood, R.H. and Guo, D. (1993). Antimutagenic potency of chlorophyllin in the Salmonella assay and its correlation with binding constants of mutagen-inhibitor complexes. Environ. Mol. Mutagen., 22, 164-171.

Guengerich, F.P. (1988). Roles of cytochrome P-450 enzymes in chemical carcinogenesis and cancer chemotherapy. Cancer Res., 48, 2946-2954.

Guengerich, F.P. and Shimada, T. (1991). Oxidation of toxic and carcinogenic chemicals by human cytochrome P-450 enzyme. Chem. Res. Toxicol., 4, 391-407.

Hashimoto, Y., Shudo, K. and Okamoto, T. (1980). Activation of mutagen, 3-amino-1-methl-5 $H$ pyrido $[4,3-b]$ indol. Identification of 3-hydroxyamino-1-methyl-5H pyrido $[4,3-b]$ indol and its reaction with DNA. Biochem. Biophys. Res. Commun., 96, 355-362.

Inoue, T., Morita, K. and Kada, T. (1981). Purification and properties of a plant desmutagenic factor for the mutagenic principle of tryptophan pyrolysate. Agric. Biol. Chem., 45, 345-353.

Kato, R. and Yamazoe, Y. (1988). $N$-hydroxyarylamine $O$-acetyltransferase in mammalian livers and Salmonella. In "Carcinogenic and Mutagenic Responses to Aromatic Amines and Nitroarenes," ed. by C. M. King, L. J. Romano and D. Schuetzie. Elsevier, Amsterdam, pp. 125-136

Miller, J.H. (1972). "Experiments in Molecular Genetics," Cold Spring Harbor Laboratory Press, New York, pp. 352-355.

Mitscher, L.A., Drake, S., Gollapudi, S.R., Harris, J.A. and Shankel, D.M. (1986). Isolation and identification of higher plant agents active in antimutagenic assay systems: Glycyrrhiza glabra. In "Antimutagenesis and Anticarcinogenesis Mechanisms," ed. by D.M. Shankel, P.E. Hartman, T. Kada and A. Hollaender. Basic Life Sciences, 39, Plenum Press, New York, pp. 153-165.

Morita, K., Hara, M. and Kada, T. (1978). Studies on natural desmutagens; Screening for vegetable and fruit factors active in inactivation of mutagenic pyrolysis products from amino acid. Agric. Biol. Chem., 42, 1235-1238.

Oda, Y., Oki, I., Kato, T. and Shinagawa, H. (1985). Evaluation of the new system ( $u m u$ system) for the detection of environmental mutagens and carcinogens. Mutat. Res., 147, 219-229.

Oda, Y., Yamazaki, H., Watanabe, M., Nohmi, T. and Shimada, T. (1995). Development of high sensitive $u m u$ test: rapid detection of genotoxicity of promutagenic aromatic amines by Salmonella typhimurium strain NM2009 possessing high $O$-acetyltransferase activity. Mutat. Res., 334, 145-156.

Okai, Y., Higashi-okai, K., Nakamura, S., Yano, Y. and Otani, S. (1996). Suppressive effects of retinoids, carotenoids and antioxidant vitamins on heterocyclic amine-induced $u m u \mathrm{C}$ gene expression in Salmonella typhimurium (TA1535/pSK 1002). Mutat. Res., 368, 133-140.

Shankel, D.M. and Clarke, C.H. (1990). Specificity of antimutagens against chemical mutagens in microbial systems. In "Antimutagenesis and Anticarcinogenesis Mechanisms II," ed. by Y. Kuroda, D.M. Shankel and M.D. Waters. Basic Life Sciences, 52, Plenum Press, New York, pp. 457-460.

Sugimura, T., Kawachi, M., Nagao, M., Yahagi, T., Seino, Y., Okamoto, T., Shudo, K., Kosuge, T., Tsuji, K., Wakabayashi, K., litaka, Y. and Itai, A. (1977). Mutagenic principles in tryptophan and phenylalanine pyrolysis products. Proc. Jpn. Acad., 53, 58-61.

Watanabe, M., Ishidate, M. and Nohmi, T. (1990). Sensitive method for the detection of mutagenic nitroarenes and aromatic amines; new derivatives of Salmonella typhimurium tester strains possessing elevated $O$-acetyltransferase levels. Mutat. Res., 234, 337-348.

Yoshikawa, K., Ishii, R., Minakata, H., Komura, H. and Kada, T. (1985). Screening of bio-antimutagens in the plant kingdom. Kinki Daigaku Kankyokagaku Kenkyusyo Kenkyuhoukoku, 13, 15-23 (in Japanese).

Yoshikawa, K. and Ishii, R. (1987). Screening of desmutagens in plants. Kinki Daigaku Kankyokagaku Kenkyusyo Kenkyuhoukoku, 15, 5-10 (in Japanese).

Yoshikawa, K., Mui, K., Ishii, R., Terashita, T., Shishiyama, J. and 
Kono, M. (1990). Desmutagenic activities of vegetables on broiled fish. Mem. Fac. Agric. Kinki Univ., 23, 55-61.

Yoshikawa, K., Inagaki, K., Murao, R., Terashita, T. and Shishiyama, J. (1991). Screening and simple methods for studying antimutagens in plants. Kinki Daigaku Kankyokagaku Kenkyusyo Kenkyuhoukoku, 19, 1-5 (in Japanese).

Yoshikawa, K., Inagaki, K., Terashita, T., Shishiyama, J., Simon, K. and Shankel, D.M. (1996a). Antimutagenic activity of extracts from Japanese eggplant. Mutat. Res., 371, 65-71.

Yoshikawa, K., Inagaki, K., Terashita, T., Shishiyama, J. and Shankel, D.M. (1996b). Desmutagenic effect of pheophytin from Japanese eggplant against several mutagens. J. Food Hyg. Soc. Jpn., 37, 295 300 . 